\title{
Constructing free actions of $p$-groups on products of spheres
}

\author{
MiCHELE KLAUS
}

\begin{abstract}
We prove that, for $p$ an odd prime, every finite $p$-group of rank 3 acts freely on a finite complex $X$ homotopy equivalent to a product of three spheres.
\end{abstract}

$57 \mathrm{~S} 17$

\section{Introduction}

The origin of the study of group actions on spheres goes back to Hopf and the spherical space form problem, which asked for a classification of finite groups that can act freely on some sphere. The first result was due to P A Smith [21], who showed that if a finite group $G$ acts freely on a sphere, then it has periodic cohomology. Later Milnor [19] gave a second necessary condition: If a finite group $G$ acts freely on a sphere, then any element in $G$ of order 2 must be central. Finally Thomas, Wall and Madsen [18] were able to prove the these two necessary conditions were in fact sufficient: a finite group $G$ acts freely on a sphere if and only if it has periodic cohomology and all involutions are central.

From the homotopy point of view, Swan [24] proved that a finite group has periodic cohomology if and only if it acts freely on a finite dimensional CW-complex homotopy equivalent to a sphere. It is a classical result that a finite group has periodic cohomology if and only if all of its abelian $p$-subgroups are cyclic. Based on that and on their own algebraic results, Benson and Carlson [6] suggested the rank conjecture: for any finite group $G$ we have that $\operatorname{rk}(G)=\operatorname{hrk}(G)$. Here $\operatorname{hrk}(G)=\min \{k \in \mathbb{N}$ | $G$ acts freely on a finite dimensional CW-complex $\left.X \simeq S^{n_{1}} \times \cdots \times S^{n_{k}}\right\}$ is the homotopy rank of $G$ and $\operatorname{rk}(G)=\max \left\{k \in \mathbb{N} \mid\right.$ there is a prime $p$ with $\left.(\mathbb{Z} / p)^{k}<G\right\}$ is the algebraic rank of $G$. With this notation, Swan's result says that $\operatorname{rk}(G)=1$ if and only if $\operatorname{hrk}(G)=1$. In the same period Heller [14] showed that $\left(\mathbb{Z}_{p}\right)^{3}$ cannot act freely on a finite dimensional $\mathrm{CW}$-complex homotopy equivalent to a product of two spheres. More recently Adem and Smith [3] showed that if $\operatorname{rk}(G)=2$ then $\operatorname{hrk}(G)=2$ for $G$ a $p$-group or $G$ a simple group (different from $\operatorname{PSL}_{3}\left(\mathbb{F}_{p}\right)$ ). Adem [2] proved also that every odd order rank two group acts freely an a finite CW-complex $X \simeq S^{n} \times S^{m}$. This also follows from a more general result of Jackson [17]. Our main theorem is the following: 
Theorem 1.1 For $p$ an odd prime, every finite $p$-group of rank 3 acts freely and cellularly on a finite $\mathrm{CW}$-complex homotopy equivalent to the product of three spheres.

A converse to Theorem 1.1 is given by Hanke [13] in the sense that if $(\mathbb{Z} / p)^{r}$ acts freely on $X=S^{n_{1}} \times \cdots \times S^{n_{k}}$ and if $p>3 \operatorname{dim}(X)$, then $r \leq k$.

We outline now the structure of the paper. Let $\mathrm{p}$ be an odd prime, let $G$ be a $p$-group and let $S(V)$ be a complex representation $G$-sphere. In Section 2 we first prove that, for all integers $k \geq 0$, there exists a positive integer $q$ such that the group $\pi_{k}\left(\operatorname{Aut}_{G}\left(S\left(V^{\oplus q}\right)\right)\right.$ ) is finite. We then incorporate this result in an outline of a known construction (see Adem [3], Connolly and Prassidis [8] and Ilhan [15]) that, in favourable conditions, gives a strategy to build group actions on products of spheres with controlled isotropy subgroups.

In Section 3 we apply Section 2 to prove that for $G$ a rank $3 p$-group, p odd, there is a free finite $G-\mathrm{CW}$-complex $X \simeq S^{m} \times S^{d} \times S^{k}$. In Section 4 we use Section 2 to generalize [3, Theorem 3.2] for a $p$-group $G$ : if $X$ is a finite dimensional $G-$ CW-complex with abelian isotropy, we show that there is a free finite dimensional $G$-CW-complex $Y \simeq X \times S^{1} \times \cdots \times S^{n_{k}}$. As a corollary we will be able to build free finite $G$-CW-complexes $X \simeq S^{n_{1}} \times \cdots \times S^{n_{\mathrm{rk}(G)}}$ for $G$ a central extension of abelian $p$-groups. Our results overlap here with those of Ûnlú and Yalçin [25].

In Section 5 we discuss the rank conjecture for some infinite groups. The motivation comes from a result of Connolly and Prassidis [8] stating that a group with finite virtual cohomological dimension that is countable and with rank 1 finite subgroups acts freely on a finite dimensional CW-complex $X \simeq S^{m}$. We show that an effective $\Gamma$-sphere does not need to exist but that the algebraic analogue still holds. In more detail, first, we define an amalgamated product $\Gamma$ such that every finite dimensional $\Gamma$-space homotopy equivalent to a sphere has an isotropy subgroup of rank 2 . Secondly, we prove that for all groups $\Gamma$ with finite virtual cohomological dimension, there is a finite dimensional $\mathbb{Z}[\Gamma]$-projective complex $\mathbf{D}$ with $H^{*}(\mathbf{D}) \cong H^{*}\left(S^{n_{1}} \times \cdots \times S^{n_{\mathrm{rk}(\Gamma)}}\right)$.

We wish to thank the referee for pointing out various mistakes and for his help in improving the presentation of the paper.

\section{The general construction}

The main result of this section is the construction of Proposition 2.7. A key ingredient of the construction is Proposition 2.5, which says that under some conditions $\pi_{k}\left(\operatorname{Aut}_{G}\left(S^{n}\right)\right)$ is finite. We begin with a series of lemmas and corollaries that we assemble into a proof of Proposition 2.5. Lemmas 2.1, 2.2 and 2.4 are individual results needed in the proof of Proposition 2.5. Lemma 2.3 serves the proof of Lemma 2.4. 
Lemma 2.1 Let $X$ be a $G-C W$-complex and let $\operatorname{Aut}_{G}(X)$ be the monoid of $G-$ equivariant self-homotopy equivalences of $X$. For each $f \in \operatorname{Aut}_{G}(X)$ we write $\operatorname{Aut}_{G}(X)_{f}$ for the path component of $f$. For $k>0$, the map of unbased homotopy classes $\varphi:\left[S^{k}, \operatorname{Aut}_{G}(X)_{f}\right] \rightarrow\left[S^{k} \times X, X\right]_{G}$ is injective and factors through:

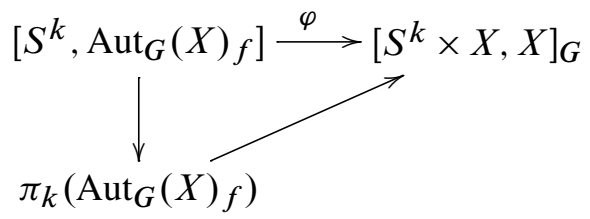

In particular all $G$-equivariant homotopies $H: I \times S^{k} \times X \rightarrow X$ between maps representing the same element in $\operatorname{Im}(\varphi)$ can be taken to satisfy $H(t, *, x)=H\left(t^{\prime}, *, x\right)$ for all $t, t^{\prime} \in I$ and $x \in X$.

Proof The map $\varphi:\left[S^{k}, \operatorname{Aut}_{G}(X)_{f}\right] \rightarrow\left[S^{k} \times X, X\right]_{G}$ is clearly well defined. To see that it is injective, consider a $G$-equivariant homotopy $H: I \times S^{k} \times X \rightarrow X$ from $\varphi\left(g_{1}\right)$ to $\varphi\left(g_{2}\right)$. Clearly $\left.H\right|_{\{0\} \times\left\{x_{0}\right\} \times X}=\varphi\left(g_{1}\right)\left(x_{0},-\right)=g_{1}\left(x_{0}\right) \in \operatorname{Aut}_{G}(X)_{f}$. Which implies that $\left.H\right|_{\{t\} \times\{x\} \times X} \in \operatorname{Aut}_{G}(X)_{f}$ for all $(t, x) \in I \times S^{k}$ because $\left.H\right|_{\{t\} \times\{x\} \times X} \simeq$ $\left.H\right|_{\{0\} \times\left\{x_{0}\right\} \times X}$ via a path in $I \times S^{k}$ from $\left(0, x_{0}\right)$ to $(t, x)$. As a result, $H$ defines an homotopy from $g_{1}$ to $g_{2}$. To prove that $\varphi$ factors through

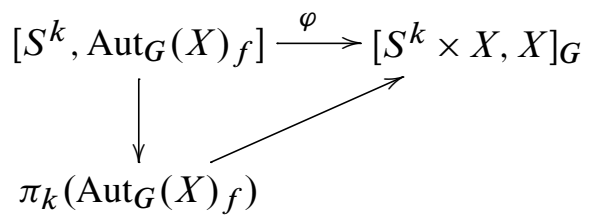

we want to show that the map $\pi_{k}\left(\operatorname{Aut}_{G}(X)_{f}\right) \rightarrow\left[S^{k}, \operatorname{Aut}_{G}(X)_{f}\right]$ is a bijection. Observe that $\operatorname{Aut}_{G}(X)$ is a monoid, thus an $H$-space so that $\pi_{1}\left(\operatorname{Aut}_{G}(X)_{\text {Id }}\right)$ acts trivially on $\pi_{k}\left(\operatorname{Aut}_{G}(X)_{\text {Id }}\right)$. The monoid $\operatorname{Aut}_{G}(X)$ is very nice because all of its connected components are homotopy equivalent through maps of the form $\operatorname{Aut}_{G}(X)_{\mathrm{Id}} \rightarrow$ $\operatorname{Aut}_{G}(X)_{f}$ with $g \mapsto f \circ g$. Thus $\pi_{1}\left(\operatorname{Aut}_{G}(X)_{f}\right)$ acts trivially on $\pi_{k}\left(\operatorname{Aut}_{G}(X)_{f}\right)$ for all $f \in \operatorname{Aut}_{G}(X)$. We conclude that $\pi_{k}\left(\operatorname{Aut}_{G}(X)_{f}\right) \rightarrow\left[S^{k}, \operatorname{Aut}_{G}(X)_{f}\right]$ is a bijection. The last claim directly follows from the diagram.

Lemma 2.2 Let $G$ be a finite group acting on a space $X$. Let $H_{1}<G$ be an isotropy subgroup maximal among isotropy subgroups. Set $X_{1}=\left\{x \in X \mid G_{x} \in\left(H_{1}\right)\right\}$, where $\left(H_{1}\right)$ denotes the conjugacy class of $H_{1}$. Then $\operatorname{Aut}_{G}\left(X_{1}\right) \cong \operatorname{Aut}_{W H_{1}}\left(X^{H_{1}}\right)$, where $W H_{1}=N H_{1} / H_{1}$ is the Weil group. 
Proof Let's begin by studying $X_{1}$. Clearly $X_{1} \subset \bigcup_{H \in\left(H_{1}\right)} X^{H}$. Since $H \in\left(H_{1}\right)$ is supposed to be maximal, we must have that if $x \in X^{H}$, then $G_{X}=H$ so that $X_{1}=\bigcup_{H \in\left(H_{1}\right)} X^{H}$. Similarly, if $x \in X^{H} \cap X^{H^{\prime}}$, for $H$ and $H^{\prime}$ in $\left(H_{1}\right)$, then $H=G_{X}=H^{\prime}$. As a result $X_{1}=\bigsqcup_{H \in\left(H_{1}\right)} X^{H}$.

Observe next that a $G$-equivariant map $f: X_{1} \rightarrow X_{1}$ restricts to a $W H_{1}$-equivariant map $f_{1}: X^{H_{1}} \rightarrow X^{H_{1}}$ because $W H_{1}=N H_{1} / H_{1}$ acts trivially on $X_{1}$. The same holds for a $G$-equivariant homotopy $F: I \times X_{1} \rightarrow X_{1}$, so that we have a well defined map res: $\operatorname{Aut}_{G}\left(X_{1}\right) \rightarrow \operatorname{Aut}_{W H_{1}}\left(X^{H_{1}}\right)$.

We want to show now that the map res: $\operatorname{Aut}_{G}\left(X_{1}\right) \rightarrow \operatorname{Aut}_{W H_{1}}\left(X^{H_{1}}\right)$ has an inverse given by $\operatorname{res}^{-1}(f)(x)=g f\left(g^{-1} x\right)$, where $g \in G$ is such that $g^{-1} x \in X^{H_{1}}$. We begin by showing that $\mathrm{res}^{-1}$ is well defined. For all $x \in X_{1}=\bigsqcup_{H \in\left(H_{1}\right)} X^{H}$ there is a $g \in G$ such that $x \in X^{g H_{1} g^{-1}}$, so that $g^{-1} x \in X^{H_{1}}$. Assume that $\gamma \in G$ is also such that $\gamma^{-1} x \in X^{H_{1}}$. Clearly $g H_{1} g^{-1}=H=\gamma H_{1} \gamma^{-1}$, where $x \in X^{H}$. Thus $\gamma^{-1} g H_{1} g^{-1} \gamma=H_{1}$ so that $\gamma^{-1} g \in N H_{1}$. For $f \in \operatorname{Aut}_{W H_{1}}\left(X^{H_{1}}\right)$ we then have $g f\left(g^{-1} x\right)=g g^{-1} \gamma f\left(\gamma^{-1} g g^{-1} x\right)=\gamma f\left(\gamma^{-1} x\right)$ because $f$ is $N H_{1}$-equivariant. Therefore res ${ }^{-1}$ is well defined.

Next, we show that $\operatorname{res}^{-1}(f)$ is $G$-equivariant: For $x \in X_{1}$, let again $g \in G$ be such that $g^{-1} x \in X^{H_{1}}$. For all $g_{0} \in G$ we have that $\left(g_{0} g\right)^{-1} g_{0} x \in X^{H_{1}}$. As a result $\operatorname{res}^{-1}(f)\left(g_{0} x\right)=g_{0} g f\left(\left(g_{0} g\right)^{-1} g_{0} x\right)=g_{0} g f\left(g^{-1} x\right)=g_{0} \operatorname{res}^{-1}(f)(x)$. In the same way one can check that if $f^{-1}$ is the homotopy inverse of $f$ via homotopies $H$ and $H^{-1}$, then $\operatorname{res}^{-1} f^{-1}$ is the homotopy inverse of $\operatorname{res}^{-1} f$ via homotopies res $^{-1} H$ and res $^{-1} H^{-1}$. Finally we observe that res o res ${ }^{-1}=\mathrm{Id}$ by choosing $g=1$, while res $^{-1}$ o res $=$ Id because $\left.\operatorname{res}^{-1} f\right|_{X^{H_{1}}}=f$ when $f$ is $G$-equivariant.

Lemma 2.3 Let $G$ be a finite group and $S^{n}$ a linear $G$-sphere. If $0<k<n$ then $H^{n}\left(S^{k} \times S^{n} / G,\{*\} \times S^{n} / G, \mathbb{Z}\right)$ is finite.

Proof Consider the long exact sequence of the pair $\left(S^{k} \times S^{n} / G,\{*\} \times S^{n} / G\right)$ with integer coefficients:

$$
\begin{aligned}
& H^{n-1}\left(S^{k} \times S^{n} / G\right) \longrightarrow H^{n-1}\left(\{*\} \times S^{n} / G\right) \\
& \downarrow \\
& H^{n}\left(S^{k} \times S^{n} / G,\{*\} \times S^{n} / G\right) \longrightarrow H^{n}\left(S^{k} \times S^{n} / G\right) \\
& i^{*} \\
& H^{n}\left(\{*\} \times S^{n} / G\right)
\end{aligned}
$$


It is clear that $H^{n}\left(S^{k} \times S^{n} / G,\{*\} \times S^{n} / G\right) \subset \operatorname{Ker}\left(i^{*}\right)$. But $H^{n}\left(S^{k} \times S^{n} / G\right) \cong$ $H^{n}\left(\{*\} \times S^{n} / G\right) \oplus H^{n-k}\left(\{*\} \times S^{n} / G\right)$. Therefore for $i^{*}: H^{n}\left(S^{k} \times S^{n} / G\right) \rightarrow$ $H^{n}\left(\{*\} \times S^{n} / G\right)$ we have that $\operatorname{Ker}\left(i^{*}\right) \cong H^{n-k}\left(\{*\} \times S^{n} / G\right)$. Finally, the groups $H^{n-k}\left(S^{n} / G\right)$ are finite for $0<k<n$ because $H^{n-k}\left(S^{n} / G, \mathbb{Q}\right)=0$ by the VietorisBegle Theorem.

Throughout the paper we will use the following notation: Let $S(V)$ be a linear $G-$ sphere. For all $H \leq G$, we write $n_{r}(H)$ for the integer such that $S\left(V^{\oplus r}\right)^{H}=S^{n_{r}(H)}$.

Lemma 2.4 Let $G$ be a finite group and $S(V)$ a linear $G$-sphere. For all $k>0$ there is an integer $q>0$ such that the groups

$$
H^{n_{q}(L)}\left(S^{k} \times S^{n_{q}(L)} / W L, \bigcup_{H>L} S^{k} \times S^{n_{q}(H)} / W L \cup\{*\} \times S^{n_{q}(L)} / W L, \mathbb{Z}\right)
$$

are finite for all $L$ with $n_{1}(L)>0$.

Proof Fix a subgroup $L<G$ such that $n_{1}(L)>0$. If there is $H>L$ with $n_{1}(H)=$ $n_{1}(L)$, then the required cohomology group is zero (it is of the form $H^{n_{1}(L)}(X, X, \mathbb{Z})$ ). Assume that for all $H>L$ we have $n_{1}(H)<n_{1}(L)$. In this case we want so show that we can take enough direct sums to be in the situation of Lemma 2.3.

Let $n_{r, L}=\max _{H>L}\left\{n_{r}(H)\right\}$ and $m_{r, L}=n_{r}(L)-n_{r, L}>0$. Observe that $n_{r}(H)=$ $r n_{1}(H)+(r-1)$ so that $n_{r, L}=r n_{1, L}+(r-1)$ and $m_{r, L}=n_{r}(L)-n_{r, L}=$ $r n_{1}(L)+(r-1)-\left(r n_{1, L}+(r-1)\right)=r m_{1, L}$. Therefore there is an $r=q_{L}$ big enough such that $m_{r, L}>k+2$. In other words $n_{r}(L)-k-2>n_{r, L}$. We have found an integer $q_{L}>0$ such that all the cells $\tau$ of the CW-complex $S^{n_{q_{L}}(L)}$ of dimension $\operatorname{dim}(\tau) \geq n_{q_{L}}(L)-k-2$, are also cells of the relative CW-complex $\left(S^{n_{q_{L}}(L)}, \bigcup_{H>L} S^{n_{q_{L}}(H)}\right)$.

We turn now our attention to the announced cohomology group. By our condition on the cells of $S^{n_{q_{L}}(L)}$, we have that the cells $\tau$ of the CW-complex $S^{k} \times S^{n_{q_{L}}(L)} / W L$ of dimension $\operatorname{dim}(\tau) \geq n_{q_{L}}(L)-2$ are also cells of the relative CW-complex

$$
\left(S^{k} \times S^{n_{q_{L}}(L)} / W L, \bigcup_{H>L} S^{k} \times S^{n_{q_{L}}(H)} / W L\right) .
$$

Henceforth,

$$
\begin{aligned}
H^{n_{q_{L}}(L)}\left(S^{k} \times S^{n_{q_{L}}(L)} / W L, \bigcup_{H>L} S^{k} \times S^{n_{q_{L}}(H)} / W L \cup\{*\} \times S^{n_{q_{L}}(L)}, \mathbb{Z}\right) \\
=H^{n_{q_{L}}(L)}\left(S^{k} \times S^{n_{q_{L}}(L)} / W L,\{*\} \times S^{n_{q_{L}}(L)} / W L, \mathbb{Z}\right) .
\end{aligned}
$$

This last group is finite, by virtue of Lemma 2.3. We conclude by observing that we can then set $q=\max _{L<G}\left\{q_{L}\right\}$. 
Proposition 2.5 Let $G$ be a finite $p$-group. Let $S(V)$ be a complex representation $G-$ sphere. For all integer $k \geq 0$ there exists an integer $q>0$ such that $\pi_{k}\left(\operatorname{Aut}_{G}\left(S\left(V^{\oplus q}\right)\right)\right)$ is finite.

Proof If $k=0$, the result has been proven by Ferrario [12]. Assume that $k>0$. Before explaining how the proof proceeds, we set up some notation: Choose an indexing of the conjugacy classes of isotropy subgroups $\left\{\left(H_{1}\right), \ldots,\left(H_{m}\right)\right\}$ such that if $\left(H_{j}\right)<\left(H_{i}\right)$ then $i<j$. Consider the filtration $S(V)_{1} \subset \cdots \subset S(V)_{m}=S(V)$ given by $S(V)_{i}=\left\{x \in S(V) \mid\left(G_{x}\right)=\left(H_{j}\right), j \leq i\right\}$. Observe that we have homomorphisms $R_{i}: \pi_{k}\left(\operatorname{Aut}_{G}(S(V))\right) \rightarrow \pi_{k}\left(\operatorname{Aut}_{G}\left(S(V)_{i}\right)\right)$ because $H\left(I \times S(V)_{i}\right) \subset$ $S(V)_{i}$ for all equivariant $H: I \times S(V) \rightarrow S(V)$. Similarly we have homomorphisms $S_{i}: \pi_{k}\left(\operatorname{Aut}_{G}\left(S(V)_{i}\right)\right) \rightarrow \pi_{k}\left(\operatorname{Aut}_{G}\left(S(V)_{i-1}\right)\right)$. Here is how the proof runs. Look at the commutative diagram:

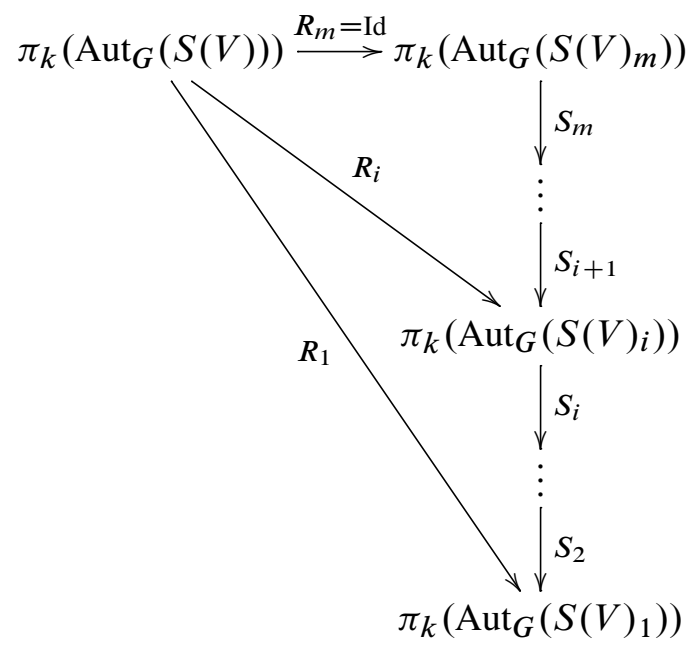

Clearly to prove that $\pi_{k}$ (Aut $\left.G(S(V))\right)$ is finite is the same as to prove that $\operatorname{Im}\left(R_{m}\right)$ is finite. To prove that $\operatorname{Im}\left(R_{m}\right)$ is finite, we will show by induction over $i$ that $\operatorname{Im}\left(R_{i}\right)$ is finite. Such an induction can be performed by showing that $\operatorname{Im}\left(R_{1}\right)$ is finite and that $S_{i}^{-1}\left(R_{i-1}(f)\right) \cap \operatorname{Im}\left(R_{i}\right)$ is finite for all $i$ and for all $f \in \pi_{k}\left(\operatorname{Aut}_{G}(S(V))\right)$. This outline can only be carried out up to replacing $S(V)$ with some power $S\left(V^{\oplus q}\right)$.

We begin by showing that there is $q_{1}>0$ such that $\pi_{k}\left(\operatorname{Aut}_{G}\left(S\left(V^{\oplus q_{1}}\right)_{1}\right)\right)$ is finite. In particular we will have that $\operatorname{Im}\left(R_{1}\right) \subset \pi_{k}\left(\operatorname{Aut}_{G}\left(S\left(V^{\oplus q_{1}}\right)_{1}\right)\right)$ is finite. For $H<G$ write $n_{r}(H)$ for the integer such that $S\left(V^{\oplus r}\right)^{H}=S^{n_{r}(H)}$. Observe that $n_{r}(H)=r n(H)+(r-1)$. By Lemma 2.2 we have that $\pi_{k}\left(\operatorname{Aut}_{G}\left(S(V)_{1}\right)\right)=$ $\pi_{k}$ (Aut $W H_{1}\left(S^{n_{1}\left(H_{1}\right)}\right)$ ). The $W H_{1}$-action on $S^{n_{1}\left(H_{1}\right)}$ is free because $H_{1}$ is maximal among isotropy subgroups. 
So Proposition 2.4 of [8] says that $\left.\pi_{k}{\text { (Aut } W H_{1}}\left(S^{n_{1}\left(H_{1}\right)}\right)\right)$ is finite if $k<n_{1}\left(H_{1}\right)-1$. If $k \geq n_{1}\left(H_{1}\right)-1$, then there is a $q_{1}>0$ for which $k<q_{1} n_{1}\left(H_{1}\right)+\left(q_{1}-1\right)-1=$ $n_{q_{1}}\left(H_{1}\right)-1$. As a result $\pi_{k}\left(\operatorname{Aut}_{G}\left(S\left(V^{\oplus q_{1}}\right)_{1}\right)\right)=\pi_{k}\left(\right.$ Aut $\left._{W H_{1}}\left(S^{n_{q_{1}}\left(H_{1}\right)}\right)\right)$ is finite (always by [8, Proposition 2.4]). Notice that the same argument also shows that $\pi_{k}\left(\operatorname{Aut}_{G}\left(S\left(V^{\oplus r q_{1}}\right)_{1}\right)\right)$ is finite for all $r>0$.

Assume that we showed that $\operatorname{Im}\left(R_{i-1}\right)$ is finite for $q \geq q_{i-1}$. The inductive step is to prove that there is $q \geq q_{i-1}$ such that $S_{i}^{-1}\left(R_{i-1}(f)\right) \cap \operatorname{Im}\left(R_{i}\right)$ is finite for all $i$ and for all $f \in \pi_{k}\left(\operatorname{Aut}_{G}\left(S\left(V^{\oplus q}\right)\right)\right)$. For that purpose we are going to use equivariant obstruction theory a la Tom Dieck [9, Section $8 ; 10$, Chapter 2]. We begin with some preliminaries. As in Lemma 2.4, let $q^{\prime}>0$ be such that the groups

$H^{n_{q^{\prime}}\left(H^{\prime}\right)}\left(S^{k} \times S^{n_{q^{\prime}}\left(H^{\prime}\right)} / W H^{\prime}, \bigcup_{H>H^{\prime}} S^{k} \times S^{n_{q^{\prime}}(H)} / W H^{\prime} \cup\{*\} \times S^{n_{q^{\prime}}\left(H^{\prime}\right)} / W H^{\prime}, \mathbb{Z}\right)$ are finite for all $H^{\prime}<G$ with $n_{1}\left(H^{\prime}\right)>0$. Let $q=\max \left\{q_{i-1}, q^{\prime}\right\}$. To simplify the notation we write $W=V^{\oplus q}, X=S^{k} \times S(W)$ and

$$
\bar{X}^{H_{i}}=\bigcup_{H>H_{i}} X^{H} \cup\{*\} \times S(W)^{H_{i}} .
$$

With this notation we have that the group

$$
H^{n_{q}\left(H_{i}\right)}\left(X^{H_{i}} / W H_{i}, \bar{X}^{H_{i}} / W H_{i}, \pi_{n_{q}\left(H_{i}\right)}\left(S^{n_{q}\left(H_{i}\right)}\right)\right)
$$

is finite by Lemma 2.4 , while if $r \neq n_{q}\left(H_{i}\right)$ then the groups

$$
H^{r}\left(X^{H_{i}} / W H_{i}, \bar{X}^{H_{i}} / W H_{i}, \pi_{r}\left(S^{n_{q}\left(H_{i}\right)}\right)\right)
$$

are finite because they are finitely generated torsion abelian groups. (The fixed points of a complex representation spheres are odd-dimensional spheres whose homotopy groups are all but one finite).

A word of explanation is in order here (see also [9, Section 8]). The space $X^{H_{i}}$ is the one over which we want to extend a map already defined on

$$
\bar{X}^{H_{i}}=\bigcup_{H>H_{i}} X^{H} \cup\{*\} \times S(W)^{H_{i}} .
$$

We have a map defined over $\bigcup_{H>H_{i}} X^{H}$ from the inductive hypothesis, while we have a map defined over $\{*\} \times S(W)^{H_{i}}$, because all the maps come from $\pi_{k}\left(\operatorname{Aut}_{G}(S(W))\right)$ in the following way: By Lemma 2.1 there is an injection $\pi_{k}\left(\operatorname{Aut}_{G}\left(S(W)_{i}\right)\right) \rightarrow$ $\left[S^{k} \times S(W)_{i}, S(W)_{i}\right]_{G}$ yielding a commutative diagram with injective columns:

$$
\begin{aligned}
& \pi_{k}\left(\operatorname{Aut}_{G}\left(S(W)_{i}\right)\right) \stackrel{S_{i}}{\longrightarrow} \pi_{k}\left(\operatorname{Aut}_{G}\left(S(W)_{i-1}\right)\right) \\
& \begin{array}{cc}
\varphi_{i} \downarrow & \varphi_{i-1} \downarrow \\
{\left[X_{i}, S(W)_{i}\right]_{G} \stackrel{s_{i}}{\longrightarrow}\left[X_{i-1}, S(W)_{i-1}\right]_{G}}
\end{array}
\end{aligned}
$$


To prove that $S_{i}^{-1}\left(R_{i-1}(f)\right) \cap \operatorname{Im}\left(R_{i}\right)$ is finite, it is enough to prove that the intersection $s_{i}^{-1}\left(\varphi_{i-1}\left(R_{i-1}(f)\right)\right) \cap \varphi_{i}\left(\operatorname{Im}\left(R_{i}\right)\right)$ is finite. By abuse of notation we will keep on writing $S_{i}$ and $R_{i-1}(f)$, but we will think of them as living in the bottom row of the diagram. Now, suppose that $g \in \pi_{k}\left(\operatorname{Aut}_{G}(S(W))\right)$ is such that there is a homotopy $h$ between $R_{i-1}(f)$ and $R_{i-1}(g)$. Such a homotopy is constant over $\{*\} \times S(W)_{i-1}$ where it coincides with both $R_{i-1}(f)$ and $R_{i-1}(g)$. Consequently, $h$ can be extended to a homotopy from $\left.R_{i}(f)\right|_{X_{i-1} \cup\{*\} \times S(W)_{i}}$ to $\left.R_{i}(g)\right|_{X_{i-1} \cup\{*\} \times S(W)_{i}}$. (Since $S^{k}$ is a trivial $G$-sphere, we have $\left.X_{i-1}=S^{k} \times S(W)_{i-1}\right)$.

Henceforth, writing $X_{i-1} \cup S(W)_{i}$ for $X_{i-1} \cup\{*\} \times S(W)_{i}$, we can apply equivariant obstruction theory inductively over $r$ to each of the diagrams:

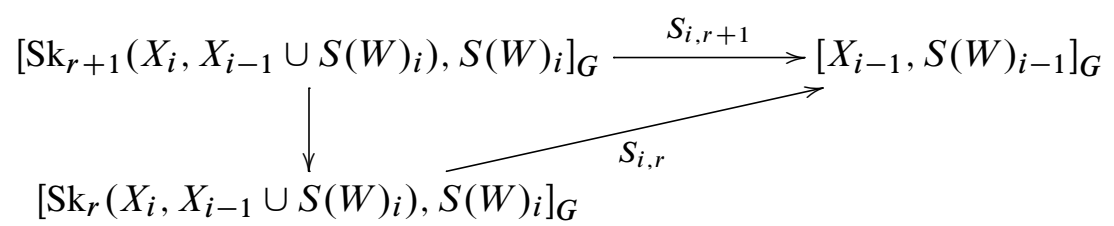

Before doing so, observe that we have restrictions

$$
\left[X_{i}, S(W)_{i}\right]_{G} \rightarrow\left[\operatorname{Sk}_{r}\left(X_{i}, X_{i-1} \cup S(W)_{i}\right), S(W)_{i}\right]_{G} .
$$

Since $\operatorname{Im}\left(R_{i}\right) \subset\left[X_{i}, S(W)_{i}\right]_{G}$, we can consider the image of $\operatorname{Im}\left(R_{i}\right)$ under these restriction maps, and we denote it by $\operatorname{Sk}_{r}\left(\operatorname{Im}\left(R_{i}\right)\right)$.

Now, If $r=0$, then $\mathrm{Sk}_{0}\left(X_{i}, X_{i-1} \cup S(W)_{i}\right)=X_{i-1} \bigsqcup\left\{x_{0}, \ldots, x_{l}\right\}$. Consequently, $S_{i, 0}^{-1}\left(R_{i-1}(f)\right) \cap \mathrm{Sk}_{0}\left(\operatorname{Im}\left(R_{i}\right)\right)$ depends on the connected components of $S(W)_{i}$. But $S(W)_{i-1}$ has finitely many connected components because it is a finite CWcomplex, therefore $S_{i, 0}^{-1}\left(R_{i-1}(f)\right) \cap \mathrm{Sk}_{0}\left(\operatorname{Im}\left(R_{i}\right)\right)$ is finite. From now on, to simplify the notation, we will write $f_{i}=R_{i}(f)$ for all possible $i$ and $f$. Assume that $S_{i, r}^{-1}\left(f_{i-1}\right) \cap \operatorname{Sk}_{r}\left(\operatorname{Im}\left(R_{i}\right)\right)=\left\{g_{i, r}^{1}, \ldots, g_{i, r}^{t}\right\}$ is finite of order $t$ (ie $g_{i, r}^{j} \neq g_{i, r}^{l}$ if $j \neq l)$. For each $g_{i, r+1} \in S_{i, r+1}^{-1, r}\left(f_{i-1}\right) \cap \operatorname{Sk}_{r+1}\left(\operatorname{Im}\left(R_{i}\right)\right)$ there is a unique $g_{i, r}^{j}$ and a homotopy $h$ from $\left.g_{i, r} \stackrel{i, r+1}{=} g_{i, r+1}\right|_{\mathrm{Sk}_{r}\left(X_{i}, X_{i-1} \cup S(W)_{i}\right)}$ to $g_{i, r}^{j}$ Notice that, by definition, we have $g^{j}, g \in \pi_{k}\left(\operatorname{Aut}_{G}(S(W))\right)$ with $g_{i, r+1}=\left.g_{i}\right|_{\mathrm{Sk}_{r+1}\left(X_{i}, X_{i-1} \cup S(W)_{i}\right)}$ and $g_{i, r}^{j}=\left.g_{i}^{j}\right|_{\operatorname{Sk}_{r}\left(X_{i}, X_{i-1} \cup S(W)_{i}\right)}$.

We write $\bar{d}\left(g_{i, r+1}, h, \mathrm{Sk}_{r+1}\left(g_{i}^{j}\right)\right) \in H^{r+1}\left(X^{H_{i}} / W H_{i}, \bar{X}^{H_{i}} / W H_{i}, \pi_{r+1}\left(\left(S^{n}\right)^{H_{i}}\right)\right)$ for the class of the difference cocycle as in [9, Section 8; 10, Chapter 2]. The element $d\left(g_{i, r+1}, h, \operatorname{Sk}_{r+1}\left(g_{i}^{j}\right)\right)$ is a cocycle because $g^{j}, g \in \pi_{k}\left(\operatorname{Aut}_{G}(S(W))\right)$ (see [10, Lemma 3.14]). The properties of the difference cocycle are given in chapter 2 of [10]. In particular we have that, if $\bar{d}\left(g_{i, r+1}^{\prime}, h^{\prime}, \operatorname{Sk}_{r+1}\left(g_{i}^{j}\right)\right)=\bar{d}\left(g_{i, r+1}, h, \operatorname{Sk}_{r+1}\left(g_{i}^{j}\right)\right)$, 
then

$$
\begin{aligned}
\bar{d}\left(g_{i, r+1}^{\prime}, h^{\prime}+h^{-1},\right. & \left.g_{i, r+1}\right) \\
& =\bar{d}\left(g_{i, r+1}^{\prime}, h^{\prime}, \mathrm{Sk}_{r+1}\left(g_{i}^{j}\right)\right)+\bar{d}\left(\mathrm{Sk}_{r+1}\left(g_{i}^{j}\right), h^{-1}, g_{i, r+1}\right) \\
& =\bar{d}\left(g_{i, r+1}^{\prime}, h^{\prime}, \mathrm{Sk}_{r+1}\left(g_{i}^{j}\right)\right)-\bar{d}\left(g_{i, r+1}, h, \mathrm{Sk}_{r+1}\left(g_{i}^{j}\right)\right)=0,
\end{aligned}
$$

so that $g_{i, r+1} \simeq g_{i, r+1}^{\prime}$. We can therefore define an injection

$$
\begin{aligned}
\bar{d}: S_{i, r+1}^{-1}\left(f_{i-1}\right) \cap & \operatorname{Sk}_{r+1}\left(\operatorname{Im}\left(R_{i}\right)\right) \\
& \rightarrow \coprod_{j=1}^{t}\left\{\left(g_{i, r}^{j}\right)\right\} \times H^{r+1}\left(X^{H_{i}} / W H_{i}, \bar{X}^{H_{i}} / W H_{i}, \pi_{r+1}\left(S^{n_{q}\left(H_{i}\right)}\right)\right)
\end{aligned}
$$

by setting $g_{i, r+1} \mapsto\left\{g_{i, r}^{j}\right\} \times \bar{d}\left(g_{i, r+1}, h, \mathrm{Sk}_{r+1}\left(g_{i}^{j}\right)\right)$. Since we chose the integer $q$ in order to have all the cohomology groups on the right hand side to be finite, we must have that the left hand side is finite as well. Summarizing, by induction we have that $S_{i, r+1}^{-1}\left(f_{i-1}\right) \cap \operatorname{Sk}_{r+1}\left(\operatorname{Im}\left(R_{i}\right)\right)$ is finite for all $r$. Since $X$ is finite dimensional, this shows that $S_{i}^{-1}\left(f_{i-1}\right) \cap \operatorname{Im}\left(R_{i}\right)$ is finite. We conclude as explained in the outline at the beginning of this proof.

We introduce next the following notation:

Notation 2.6 Let $G$ be a finite group and $X$ a $G-C W-$ complex. We write

$$
\operatorname{rk}_{X}(G)=\max \left\{n \in \mathbb{N} \mid \text { there exists } G_{\sigma} \text { with } \operatorname{rk}\left(G_{\sigma}\right)=n\right\} .
$$

We can now state the main result of the section:

Proposition 2.7 Let $G$ be a finite $p$-group and let $X$ be a finite dimensional $G-C W$ complex. Assume that to each isotropy subgroup $G_{\sigma}$ we can associate a representation $\rho_{\sigma}: G_{\sigma} \rightarrow U(n)$ such that $\left.\rho_{\sigma}\right|_{G \tau} \cong \rho_{\tau}$ whenever $G_{\tau}<G_{\sigma}$. If $\rho_{\sigma}$ is fixed point free for all $H \leq G_{\sigma}$ with $\operatorname{rk}(H)=\operatorname{rk}_{X}(G)$, then there exists a finite dimensional $G-C W-$ complex $E \cong X \times S^{m}$ with $\operatorname{rk}_{E}(G)=\operatorname{rk}_{X}(G)-1$. Moreover, if $X$ is finite then $E$ is finite as well.

Proof The proof follows [8]. We refer the reader to [15] for the details. Write $S_{\sigma}^{2 n-1}$ for the linear sphere associated to $\rho_{\sigma}$. We want to glue these spheres into a $G-$ equivariant spherical fibration over $X$. We will proceed by induction over the skeleton of $X$. For every $G$-orbit of the 0 -skeleton, choose a representative $\sigma$ and define a map $G \times{ }_{G_{\sigma}} S_{\sigma}^{2 n-1} \rightarrow X^{0}$ by $(g, x) \mapsto g \cdot \sigma$. This defines a $G$-equivariant spherical 
fibration $S^{2 n-1} \rightarrow E_{0} \rightarrow \mathrm{Sk}_{0}(X)$ whose total space is a finite dimensional $G-\mathrm{CW}-$ complex. Clearly if $\rho_{\sigma}$ is fixed point free for all $H \leq G_{\sigma}$ with $\operatorname{rk}(H)=\operatorname{rk}_{X}(G)$, then $\operatorname{rk}\left(E_{0}\right)=\operatorname{rk}_{X}(G)-1$.

The inductive step is next. Suppose given a $G$-equivariant spherical fibration over the $(k-1)$-skeleton $*^{q_{k-1}} S^{2 n-1} \rightarrow E_{k-1} \rightarrow \operatorname{Sk}_{k-1}(X)$ whose total space is a finite dimensional $G-\mathrm{CW}$-complex. Assume also that if $\rho_{\sigma}$ is fixed point free for all $H \leq G_{\sigma}$ with $\operatorname{rk}(H)=\operatorname{rk}_{X}(G)$, then $\operatorname{rk}\left(E_{k-1}\right)=\operatorname{rk}_{X}(G)-1$. Now, for every $G$-orbit of a $k$-cell, choose a representative $\sigma$. The $G_{\sigma}$-equivariant fibration $*^{q_{k-1}} S^{2 n-1} \rightarrow$ $\left.E_{k-1}\right|_{\partial \sigma} \rightarrow \partial \sigma$ is classified by an element $a_{\sigma} \in \pi_{k-2}\left(\operatorname{Aut}_{G_{\sigma}}\left(*^{q_{k-1}} S^{2 n-1}\right)\right)$.

We want to have $a_{\sigma}=0$ : Observe that, in general, for two complex $G$-spheres $S(V)$ and $S(W)$, we have that $S(V \oplus W) \cong S(V) * S(W)$ as $G$-spheres. Therefore, by Proposition 2.5, we can take enough Whitney sums of the fibration $*^{q_{k-1}} S^{2 n-1} \rightarrow$ $E_{k-1} \rightarrow \operatorname{Sk}_{k-1}(X)$ to guarantee that $a_{\sigma}=0$ (see [8, Lemma 2.3, Proposition 2.4]). We can then extend the $G_{\sigma}$-equivariant fibration $\left.*^{q_{k}} S^{2 n-1} \rightarrow E_{k-1}\right|_{\partial \sigma} \rightarrow \partial \sigma$ equivariantly across the cell $\sigma$. We define a $G$-equivariant spherical fibration over the orbit of $\sigma$ by $G \times{ }_{G_{\sigma}} *^{q_{k}} S_{\sigma}^{2 n-1} \rightarrow G \sigma$ with $(g, x) \mapsto g \cdot \sigma$.

Repeating the procedure for all the representatives of the $G$-orbits of $k$-cells, we recover a $G$-equivariant spherical fibration ${ }^{q_{k}} S^{2 n-1} \rightarrow E_{k} \rightarrow \operatorname{Sk}_{k}(X)$ with total space a finite dimensional $G-\mathrm{CW}$-complex. Clearly if $\rho_{\sigma}$ is fixed point free for all $H \leq G_{\sigma}$ with $\operatorname{rk}(H)=\operatorname{rk}_{X}(G)$, then $\operatorname{rk}\left(E_{k}\right)=\operatorname{rk}_{X}(G)-1$. We conclude noticing that, by Proposition 2.8 in [3], up to taking further fibre joins, we can assume that the total fibration $*^{q} S^{2 n-1} \rightarrow E \rightarrow X$ is a product one.

For the last statement, one can observe that all the constructions take place in the category of finite CW-complexes, providing that the initial space $X$ is a finite $\mathrm{CW}-$ complex.

\section{Rank 3 -groups, $p$ odd}

The results of this section have also been announced by Jackson [16]. We give a proof which uses the group theory developed there. For the convenience of the reader we reproduce it here.

Lemma 3.1 If $G$ is a finite $p$-group with $\operatorname{rk}(G)=3$ and $\operatorname{rk}(Z(G))=1$, then there exists a normal abelian subgroup $Q<G$ of type $(p, p)$ with $Q \cap Z(G) \neq 0$.

Proof This is proven by Suzuki [23, Section 4]. 
Proposition 3.2 Let $G$ be a finite $p$-group with $p>2, \operatorname{rk}(G)=3$ and $\operatorname{rk}(Z(G))=1$. Let $Q$ be an abelian normal subgroup of type $(p, p)$ as above. Suppose that $H<G$ with $H \cap Z(G)=0$ and $|H|=p^{n}$. Then either $H$ is cyclic, $H<C_{G}(Q), H$ is abelian of type $\left(p, p^{n-1}\right)$ or $H \cong M\left(p^{n}\right)=\left\langle x, y \mid x^{p^{n-1}}=y^{p}=1, y^{-1} x y=x^{1+p^{n-2}}\right\rangle$.

Proof If $\operatorname{rk}(H)=1$ then $H$ is cyclic since $p>2$. Suppose that $\operatorname{rk}(H)=2$ and $H \cap Q \neq 0$. By assumption $Z(G) \cap Q=\mathbb{Z} / p, H \cap Z(G)=0$ and $Q \cap H=\mathbb{Z} / p$. The map $c: H \rightarrow \operatorname{Aut}(H \cap Q)$ given by $c_{h}(x)=h x h^{-1}$ is well defined because $Q$ is normal (by Lemma 3.1). Since $|H|=p^{n}$ and $|\operatorname{Aut}(H \cap Q)|=p-1$, we have that the map $c$ is trivial. As a result we have that $H<C_{G}(Q)$.

Assume now that $H \cap Q=0$. In this case $H \cap C_{G}(Q) \neq H$ since otherwise we would have $\operatorname{rk}(G)>3$. Set $K=H \cap C_{G}(Q)$ and observe that $K$ is cyclic (else we would have $\operatorname{rk}(G)>3$ ). Assume for a moment that $\left[G: C_{G}(Q)\right]=p$. In this case $[H: K]=p$, in other words $H$ has a maximal cyclic subgroup. By [23, Section 4], $H$ needs then to be abelian of type $\left(p, p^{n-1}\right)$ or $M\left(p^{n}\right)$.

We still have to prove that $\left[G: C_{G}(Q)\right]=p$. The group $G$ acts on $Q$ by conjugation and for each element $q$ of $Q$ not in center of $G$, we have that $G_{q}=C_{G}(Q)$. As a result $\left|C_{G}(Q)\right|=\left|G_{q}\right|=|G| / p$ since $Q \cong(Z / p)^{2}$ with the first coordinate in the center $Z(G)$.

Proposition 3.3 Let $G$ be a finite $p$-group with $p>2, \operatorname{rk}(G)=3$ and $\operatorname{rk}(Z(G))=1$. There exists a class function $\beta: G \rightarrow \mathbb{C}$ such that for any subgroup $H \subset G$, with $H \cap Z(G)=0$, the restriction $\left.\beta\right|_{H}$ is a complex character of $H$. If in addition $H$ is a rank two elementary abelian subgroup, then the character $\left.\beta\right|_{H}$ corresponds to an isomorphism class of fixed-point free representations.

Proof Define $\beta: G \rightarrow \mathbb{C}$ as follows:

$$
x \mapsto \begin{cases}\left(p^{2}-p\right)|G| & \text { if } x=0, \\ 0 & \text { if } x \in Z(G) \backslash 0, \\ -p|G| & \text { if } x \in Q \backslash Z(G), \\ 0 & \text { if } x \in C_{G}(Q) \backslash Q, \\ -|G| & \text { if } x \in G \backslash C_{G}(Q) \text { of order p, } \\ 0 & \text { if } x \in G \backslash C_{G}(Q) \text { of order greater than } \mathrm{p} .\end{cases}
$$

The map $\beta$ is a class function because we have the following sequence of subgroups each normal in $G$ :

$$
0<Z(G)<Q<C_{G}(Q)<G
$$


For the sequel of the proof, we set

$$
\phi_{k}: \mathbb{Z} / p^{n} \rightarrow \mathbb{C}, \quad x \mapsto e^{2 \pi i k x / n},
$$

while

$$
\phi_{k} \phi_{j}: \mathbb{Z} / p^{n} \times \mathbb{Z} / p^{n} \rightarrow \mathbb{C}, \quad(x, y) \mapsto e^{2 \pi i(k x+j y) / n} .
$$

To lighten the notation, we drop the dependence in $n$ on the $\phi_{k}$ 's because it will be clear from the context. To understand correctly the characters that follow, it will be important to specify the generators of the elementary abelian subgroups treated. Consider first an elementary abelian subgroup $H$ of $G$ of rank 2 and which intersects trivially the center $Z(G)$. If $H \cap Q \neq 0$, then $H \cap Q \cong \mathbb{Z} / p$ and $H \cong(H \cap Q) \times \mathbb{Z} / p$ so that

$$
\left.\beta\right|_{H}=|G| \sum_{k=0}^{p-1} \sum_{j=1}^{p-1} \phi_{k} \phi_{j}
$$

If $H \cap Q=0$, then $\mathbb{Z} / p \cong H \cap C_{G}(Q)$ so that $H \cong\left(H \cap C_{G}(Q)\right) \times \mathbb{Z} / p$ and

$$
\left.\beta\right|_{H}=(p-1)|G| / p\left(\sum_{k=0}^{p-1} \sum_{j=1}^{p-1} \phi_{j} \phi_{k}\right)+|G| \sum_{k=1}^{p-1} \phi_{0} \phi_{k} .
$$

Consider now a subgroup $H$ of $G$ with $H \cap Z(G)=0$. We will proceed case by case using the classification above.

(1) If $H \cap Q \neq 0$ then $H \subset C_{G}(Q)$ and $|K|=p$ with $K=Q \cap H$. Let $\phi$ be the character of $K$ which is $p-1$ on the identity and -1 for each other element of $K$. Then

$$
\left.\beta\right|_{H}=\frac{p|G|}{|H: K|} \operatorname{Ind}_{K}^{H} \phi .
$$

(2) If $H \cap Q=0$ and $H \subset C_{G}(Q)$ then $H$ is cyclic and $\left.\beta\right|_{H}=\left(p^{2}-p\right)|G| /|H| \phi$ where $\phi$ is the character of $H$ that is $|H|$ on the identity and 0 elsewhere.

(3) If $H \cap Q=0$ and $H$ is cyclic with $H \cap C_{G}(Q)=0$, then $|H|=p$ and $\left.\beta\right|_{H}=|G| / p \phi$ where $\phi$ is $\left(p^{3}-p^{2}\right)$ on the identity and $-p$ elsewhere.

(4) If $H \cap Q=0$ and $H$ is cyclic with $H \cap C_{G}(Q) \neq 0$, then

$$
\left.\beta\right|_{H}=\left(p^{2}-p\right)|G| /|H| \sum_{k=0}^{|H|-1} \phi_{k} .
$$

(5) Assume that $H \cap Q=0$ and that $H$ is abelian of type $\left(p, p^{n-1}\right)$. Write $H=$ $\left\langle x, y \in H \mid x^{p}=y^{p^{n-1}}=1,[x, y]=1\right\rangle$. Notice that $\langle y\rangle=H \cap C_{G}(Q)$. For each $1 \leq i \leq p$ set $H_{i}=\left\langle x y^{i p^{n-2}}\right\rangle$. Clearly $\left|H_{i}\right|=p, H_{i} \cap C_{G}(Q)=0$ and $H_{i} \cap H_{j}=0$ if $i \neq j$. 
Let $\eta_{i}$ be the character of $H_{i}$ which is $p-1$ on the identity and -1 elsewhere. Set

$$
\phi=\sum_{i=1}^{p} \operatorname{Ind}_{H_{i}}^{H} \eta_{i} .
$$

Since $\phi(1)=|H|(p-1), \phi(z)=-|H| / p$ for $z \in H \backslash\langle y\rangle$ and $\phi(z)=0$ for $z \in\langle y\rangle$; we conclude that $\left.\beta\right|_{H}=p|G| /|H| \phi$.

(6) If $H \cap Q=0$ and $H \cong M\left(p^{n}\right)$, we can write

$$
H=\left\langle x, y \mid x^{p^{n-1}}=y^{p}=1, y^{-1} x y=x^{1+p^{n+2}}\right\rangle .
$$

Let $N=\left\langle x^{p^{n-2}}, y\right\rangle \cong(\mathbb{Z} / p)^{2}$ which is normal in $H$. Let $\phi$ be the character of $N$ given by

$$
\phi=(p-1)\left(\sum_{k=0}^{p-1} \sum_{j=1}^{p-1} \phi_{j} \phi_{k}\right)+p \sum_{k=1}^{p-1} \phi_{0} \phi_{k} .
$$

Then we have $\phi(0)=p^{2}(p-1), \phi\left(\left(x^{k p^{n-2}}, 0\right)\right)=0$, while $\phi\left(\left(x^{k p^{n-2}}, y^{l}\right)\right)=-p$. Finally $\left.\beta\right|_{H}=\operatorname{Ind}_{N}^{H}|G| / p|H: N| \phi$.

We can now turn our attention to the topological problem:

Proposition 3.4 For every odd order rank $3 p$-group $G$, there is a finite dimensional $G-C W$-complex $X \simeq S^{m} \times S^{n}$ with cyclic isotropy subgroups.

Proof Suppose first that $Z(G)$ is not cyclic. Fix a subgroup $\mathbb{Z} / p \times \mathbb{Z}_{p}<Z(G)$. Obviously, there are two complex representations $\rho_{1}: \mathbb{Z} / p \times \mathbb{Z} / p \rightarrow \operatorname{GL}\left(V_{1}\right)$ and $\rho_{2}: \mathbb{Z} / p \times \mathbb{Z} / p \rightarrow \operatorname{GL}\left(V_{2}\right)$, such that $\mathbb{Z} / p \times \mathbb{Z} / p$ acts freely on $S\left(V_{1}\right) \times S\left(V_{2}\right)$. Consider $\eta_{1}=\operatorname{Ind}_{\mathbb{Z} / p \times \mathbb{Z} / p}^{G} \rho_{1}: G \rightarrow \operatorname{GL}\left(W_{1}\right)$ and $\eta_{2}=\operatorname{Ind}_{\mathbb{Z} / p \times \mathbb{Z} / p}^{G} \rho_{2}: G \rightarrow \operatorname{GL}\left(W_{2}\right)$. The $G$-space $S\left(W_{1}\right) \times S\left(W_{2}\right)$ has rank 1 isotropy groups. Since $p$ is odd, this means that the isotropy groups are cyclic.

Assume that $Z(G)$ is cyclic and let $S^{m}$ be the linear $G$-sphere obtained by inducing from a free linear action of $Z(G)$. The isotropy subgroups for this action are the one described in Proposition 3.2. The conditions of Proposition 2.7 are fulfilled by Proposition 3.3. The conclusion follows.

As a direct consequence of [3, Theorem 3.2] we obtain:

Theorem 3.5 For every odd order rank 3 -group $G$, there is a free finite $G-C W$ complex $X \cong S^{m} \times S^{n} \times S^{k}$. 
Note that a converse to Theorem 3.5 is given by Hanke in [13] in the sense that if $(\mathbb{Z} / p)^{r}$ acts freely on $X=S^{n_{1}} \times \cdots \times S^{n_{k}}$ and if $p>3 \operatorname{dim}(X)$, then $r \leq k$.

Remark For $p=2$ the situation is more complicated because of the classification of subgroups. A 2-group of rank 1 can be either cyclic or generalized quaternion. A 2group with a maximal abelian subgroup can be cyclic, generalized quaternion, dihedral, $M\left(2^{n}\right)$ (see Proposition 3.2) or $S_{4 m}=\left\langle x, y \mid x^{2 m}=y^{2}=1, y^{-1} x y=x^{m-1}\right\rangle$ (see [23, Section 4, Chapter 4]). For $p=2$, the class function of Proposition 3.3 does not restrict to characters over the subgroups, in general.

\section{Abelian isotropy $p$-groups}

We begin by generalizing [3, Theorem 3.2] for $p$-groups.

Theorem 4.1 Let $G$ be a finite $p$-group and let $X$ be a finite dimensional $G-C W-$ complex with $G_{\sigma}$ abelian for all cells $\sigma \subset X$. Then there is a free finite dimensional $G-C W$-complex $Y \simeq X \times S^{n_{1}} \times \cdots \times S^{\mathrm{rk}_{X}(G)}$. Moreover, if $X$ is finite, then $Y$ is finite as well.

Proof We prove the theorem by induction over $\operatorname{rk}_{X}(G)$. If $\operatorname{rk}_{X}(G)=1$, the theorem has been proven by Adem and Smith [3, Theorem 3.2].

The inductive step follows. By virtue of Proposition 2.7, we only need to associate to each isotropy subgroup $G_{\sigma}$ a representation $\rho_{\sigma}: G_{\sigma} \rightarrow U(m)$ such that $\left.\rho_{\sigma}\right|_{G \tau} \cong \rho_{\tau}$ whenever $G_{\tau}<G_{\sigma}$ and such that $\rho_{\sigma}$ is fixed point free for all $H \leq G_{\sigma}$ with $\operatorname{rk}(H)=\operatorname{rk}_{X}(G)$.

Consider the class function $\beta: G \rightarrow \mathbb{C}$ given by

$$
x \mapsto \begin{cases}|G|\left(p^{\mathrm{rk}_{X}(G)}-1\right) & \text { if } x=0, \\ -|G| & \text { if } o(x)=p, \\ 0 & \text { otherwise. }\end{cases}
$$

To simplify the notation write $A=G_{\sigma}$ for an isotropy subgroup (which is abelian by hypothesis). We need to prove that $\left.\beta\right|_{A}$ is a character which is fixed point free for all $(\mathbb{Z} / p)^{\mathrm{rk} X}(G) \leq A$. Set $A_{p}=\{0\} \cup\{x \in A \mid o(x)=p\}$. Since $A$ is abelian we have $A_{p} \triangleleft A$. Fix an injection $f: A_{p} \rightarrow(\mathbb{Z} / p)^{\mathrm{rk}_{X}(G)}$. Write $\rho_{0}:(\mathbb{Z} / p)^{\mathrm{rk}_{X}(G)} \rightarrow$ $U\left(p^{\mathrm{rk}_{X}(G)}-1\right)$ for the reduced regular representation and let $\rho=\rho_{0} \circ f$ be the representation $A_{p} \rightarrow(\mathbb{Z} / p)^{\mathrm{rk}_{X}(G)} \rightarrow U\left(p^{\mathrm{rk}_{X}(G)}-1\right)$. Consider finally the representation of $A$ given by $\eta=|G|\left|A_{p}\right| /|A| \operatorname{Ind}_{A_{p}}^{A} \rho$. Clearly $\eta(0)=|G|\left(p^{\mathrm{rk}_{X}(G)}-1\right)$, $\eta(x)=-|G|$ if $x \in A_{p} \backslash 0$ while $\eta(x)=0$ if $x \notin A_{p}$. As a result $\left.\beta\right|_{A}=\eta$. If now 
$(\mathbb{Z} / p)^{\mathrm{rk}_{X}(G)} \leq A$, then $(\mathbb{Z} / p)^{\mathrm{rk}_{X}(G)} \leq A_{p}$. Consequently $\left.\eta\right|_{(\mathbb{Z} / p)^{\mathrm{rk} X}(G)}$ is a multiple of the reduced regular representation, thus fixed point free.

Corollary 4.2 Let $G$ be a $p$-group. Assume that $G$ is a central extension of abelian groups, then there is a free finite $G-C W$-complex $X \simeq S^{n_{1}} \times \cdots \times S^{n_{\mathrm{rk}(G)}}$. The result in particular holds for extraspecial $p$-groups.

Proof Let $X=S^{n_{1}} \times \cdots \times S^{n_{\mathrm{rk}}(Z(G))}$ be the product of the $G$-spheres arising from suitable representations of the center. Clearly $\operatorname{rk}_{X}(G)=\operatorname{rk}(G)-\operatorname{rk}(Z(G))$ and $G_{\sigma}$ is abelian. The conclusion follows.

\section{Infinite groups}

As pointed out in [8], there is a class of infinite groups which is worth considering, when studying the rank conjecture mentioned in the introduction (which is usually stated for finite groups). This is the class of groups $\Gamma$ of finite virtual cohomological dimension. Recall that, by definition, a group $\Gamma$ has finite virtual cohomological dimension, if it has a finite index subgroup $\Gamma^{\prime}<\Gamma$ with finite cohomological dimension (that is to say, $H^{n}\left(\Gamma^{\prime}, M\right)=0$ for all coefficients $M$ and for all $n$ big enough). Occasionally, we will write "finite ved" for "finite virtual cohomological dimension".

See, for example, Brown [7] for background on groups with finite virtual cohomological dimension. The crucial property that makes them interesting to us is the following: for any such group $\Gamma$ there exists a finite dimensional $\Gamma$-CW-complex $\mathfrak{E} \Gamma$ with $\left|\Gamma_{x}\right|<\infty$ for all $x \in \mathfrak{E} \Gamma$.

It is already known that a group with finite virtual cohomological dimension, which is countable and with rank at most 1 finite subgroups, acts freely on a finite dimensional CW-complex $X \simeq S^{m}$ [8]. The next step would be to prove the analogue result for groups $\Gamma$ with rank at most 2 finite subgroups. The easiest examples to consider are amalgamated products $\Gamma=G_{1} * G_{0} G_{2}$, where $G_{i}$ is a finite group for $i=0,1,2$ and $G_{0}<G_{i}$ for $i=1,2$.

In this case, for every finite subgroup $H<\Gamma$, there is $\gamma \in \Gamma$ such that $\gamma H \gamma^{-1}<G_{i}$ for $i=1$ or $i=2$ (see Serre [20]). In particular $\operatorname{rk}(\Gamma)=\max \left\{\operatorname{rk}\left(G_{1}\right), \operatorname{rk}\left(G_{2}\right)\right\}$. The first attempt would be to find an effective $\Gamma$-sphere, ie a $\Gamma$-sphere with rank 1 isotropy subgroups. In the first subsection we exhibit an amalgamation of two $p$-groups which doesn't have an effective $\Gamma$-sphere.

Recall from [6] that, for a finite group $G$, we have that $\operatorname{rk}(G)=r$ if and only if there are $r$ finite dimensional $\mathbb{Z}[G]$-complexes $\mathbf{K}_{1}, \ldots, \mathbf{K}_{r}$ such that $\mathbf{K}=\mathbf{K}_{1} \otimes \cdots \otimes \mathbf{K}_{r}$ 
is a complex of projective $\mathbb{Z}[G]$-modules with $H^{*}(\mathbf{K}) \cong H^{*}\left(S^{n_{1}} \times \cdots \times S^{n_{r}}\right)$. In the second subsection we prove a similar result: for every group $\Gamma$ with finite vcd, there are $\operatorname{rk}(\Gamma)$ finite dimensional $\mathbb{Z}[\Gamma]$-complexes $\mathbf{C}_{1}, \ldots, \mathbf{C}_{\mathrm{rk}(\Gamma)}$ such that $\mathbf{D}=$ $C_{*}(\mathfrak{E} \Gamma) \otimes \mathbf{C}_{1} \otimes \cdots \otimes \mathbf{C}_{\mathrm{rk}(\Gamma)}$ is a complex of projective $\mathbb{Z}[\Gamma]$-modules with $H^{*}(\mathbf{D}) \cong$ $H^{*}\left(S^{n_{1}} \times \cdots \times S^{\left.n_{\mathrm{rk}(\Gamma)}\right)}\right.$.

As a result, the group $\Gamma$ introduced in the first subsection here below satisfies the algebraic analogue of the rank conjecture but doesn't have an effective $\Gamma-$ sphere. The geometric problem of knowing whether or not $\Gamma$ acts freely on a product of two spheres is still open.

\subsection{A group without effective action on a sphere}

Let $E$ and $E^{\prime}$ be two copies of the extraspecial $p$-group of order $p^{3}$ and exponent $p$. (Such a group can be identified with the upper triangular $3 \times 3$ matrices over $\mathbb{F}_{p}$ with 1 on the diagonal). Consider the amalgamated product $\Gamma=E^{\prime} * \mathbb{Z} / p E$ given by $\mathbb{Z} / p=Z(E)$ and an injective map $f: \mathbb{Z} / p \rightarrow E^{\prime}$ with $f(\mathbb{Z} / p) \cap Z\left(E^{\prime}\right)=1$. Clearly $\operatorname{rk}(\Gamma)=2$. Let $\Gamma$ act on a finite dimensional CW-complex $X \simeq S^{n}$. Consider the restriction of this action to $E$ and $E^{\prime}$. It is well known that the dimension function of a $p$-group action on a sphere is realized by a representation over the real numbers by Dotzel and Hamrick [11] (see the next remark). Therefore, an even multiple of the dimension functions for $E$ and $E^{\prime}$ must be realized by characters $\chi_{E}$ and $\chi_{E^{\prime}}$.

Clearly the dimension functions of $\chi_{E}$ and $\chi_{E^{\prime}}$ must agree over $Z(E)$ and $f(\mathbb{Z} / p)$. Looking at the character table of $E$, we observe that every irreducible character $\alpha$, giving rise to an effective sphere, vanishes outside $Z(E)$ while $\alpha(z)=m \zeta_{p}$ for all $z \in Z(E) \backslash\{0\}$ (here $\zeta_{p}$ is a $p$-root of the unity). Thus, $\chi_{E}$ and $\chi_{E^{\prime}}$ cannot be both characters giving rise to effective spheres. We deduce that the original action must have some finite isotropy subgroups of rank 2.

This provides an example of an infinite group, with rank 2 finite $p$-subgroups, not acting with effective Euler class on any sphere.

Remark In [11] the result is stated only for finite CW-complexes. The portion of this theorem that we need, still holds for finite dimensional CW-complexes: Let $G$ be a finite $p$-group and let $X$ be a finite dimensional $G-\mathrm{CW}$-complex. If $X$ has the $\bmod p$ homology of a sphere, then $X^{H}$ has the $\bmod p$ homology of a sphere for all $H<G$. For the case $H \cong \mathbb{Z} / p$, this has been proved in [22]. The general case can be proved by induction, noticing that $X^{H}=\left(X^{\mathbb{Z} / p}\right)^{H / \mathbb{Z} / p}$, where $\mathbb{Z} / p \triangleleft$ $H$. We therefore recover a dimension function over the subgroups of $G$, given by $\operatorname{dim}_{X}(H)=\operatorname{dim}\left(H^{*}\left(X^{H}, \mathbb{Z} / p\right)\right)$, as in the finite case. Finally, we can realize this dimension function just as in [11]. 


\subsection{Algebraic spheres}

Let $\Gamma$ be a group with finite ved and rank $r$. Recall that we want to show that there are $\operatorname{rk}(\Gamma)$ finite dimensional $\mathbb{Z}[\Gamma]$-complexes $\mathbf{C}_{1}, \ldots, \mathbf{C}_{r}$ such that

$$
\mathbf{D}=C_{*}(\mathfrak{E} \Gamma) \otimes \mathbf{C}_{1} \otimes \cdots \otimes \mathbf{C}_{r}
$$

is a complex of projective $\mathbb{Z}[\Gamma]$-modules with $H^{*}(\mathbf{D}) \cong H^{*}\left(S^{n_{1}} \times \cdots \times S^{n_{r}}\right)$.

We begin with some preliminaries concerning the cohomology of finite groups. We follow here Benson [4; 5]. Let $G$ be a finite group. Consider $\zeta \in H^{n}(G, R) \cong$ $\operatorname{Ext}_{R G}^{n}(R, R) \cong \operatorname{Hom}_{R G}\left(\widehat{\Omega}^{n} R, R\right)$, where $\widehat{\Omega}^{n} R$ is the $n$-th kernel in a $R G$-projective resolution $\mathbf{P}$ of $R$. We choose a cocycle $\hat{\zeta}: \widehat{\Omega}^{n} R \rightarrow R$ representing $\zeta$. By making $\mathbf{P}$ large enough we can assume that $\hat{\zeta}$ is surjective. We denote $L_{\zeta}$ its kernel and form the pushout diagram:

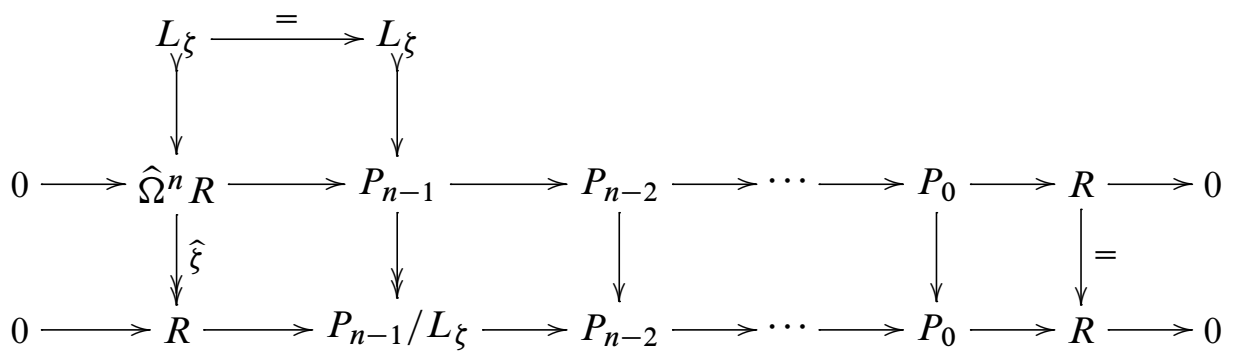

We denote by $\mathbf{C}_{\zeta}$ the chain complex

$$
0 \rightarrow P_{n-1} / L_{\zeta} \rightarrow P_{n-2} \rightarrow \cdots \rightarrow P_{0} \rightarrow R \rightarrow 0
$$

formed by truncating the bottom row of this diagram. Thus we have that $H_{0}\left(\mathbf{C}_{\zeta}\right)=$ $H_{n-1}\left(\mathbf{C}_{\zeta}\right)=R$ while $H_{i}\left(\mathbf{C}_{\zeta}\right)=0$ if $i \neq 0, n-1$. A useful result is given in the proof of [1, Theorem 3.1]:

Proposition 5.1 Let $G$ be a finite group. For all positive integer $r$, there exist classes $\xi_{1}, \ldots, \xi_{r} \in H^{*}(G, \mathbb{Z})$ such that, for all $H<G$ with $\operatorname{rk}(H) \leq r$, the complex $\mathbb{Z}[G / H] \otimes L_{\xi_{1}} \otimes \cdots \otimes L_{\xi_{r}}$ is $\mathbb{Z}[G]$-projective.

Proof See the proof of [1, Theorem 3.1].

Corollary 5.2 Let $G$ be a finite group. For all positive integer $r$, there exist $r$ finite dimensional $\mathbb{Z}[G]$-complexes $\boldsymbol{C}_{1}, \ldots, \boldsymbol{C}_{r}$ such that $H^{*}\left(\boldsymbol{C}_{1} \otimes \cdots \otimes \boldsymbol{C}_{r}\right)=$ $H^{*}\left(S^{n_{1}} \times \cdots \times S^{n_{r}}\right)$; with $\boldsymbol{C}_{1} \otimes \cdots \otimes \boldsymbol{C}_{r}$ a complex of $\mathbb{Z}[H]$-projective modules for all $H<G$ with $\operatorname{rk}(H) \leq r$. 
Proof Let $\xi_{1}, \ldots, \xi_{r} \in H^{*}(G, \mathbb{Z})$ be the classes given in Proposition 5.1. Consider the chain complex $\mathbf{C}=\mathbf{C}_{\xi_{1}} \otimes \cdots \otimes \mathbf{C}_{\xi_{r}}$. Clearly $H^{*}(\mathbf{C})=H^{*}\left(S^{n_{1}} \times \cdots \times S^{n_{r}}\right)$. For the second part of the claim, observe that all the modules in $\mathbf{C}_{\xi_{i}}$ are $\mathbb{Z}[G]$ projective except the module $P_{n_{i}-1} / L_{\xi_{i}}$. Recall that the tensor product of any module with a projective module is projective, so that it remains to examine the module $P_{n_{1}-1} / L_{\xi_{1}} \otimes \cdots \otimes P_{n_{r}-1} / L_{\xi_{r}}$. Let $H<G$ be such that $\operatorname{rk}(H) \leq r$. Since $\mathbb{Z}[G / H] \otimes L_{\xi_{1}} \otimes \cdots \otimes L_{\xi_{r}}$ is $\mathbb{Z}[G]$-projective by Proposition 5.1, we conclude that $\mathbb{Z}[G / H] \otimes P_{n_{1}-1} / L_{\xi_{1}} \otimes \cdots \otimes P_{n_{r}-1} / L_{\xi r}$ is $\mathbb{Z}[G]$-projective as in [5, Theorem 5.14.2]. It then easily follows that $P_{n_{1}-1} / L_{\xi_{1}} \otimes \cdots \otimes P_{n_{r}-1} / L_{\xi_{r}}$ is $\mathbb{Z}[H]$-projective.

We go back now to our group $\Gamma$ with finite ved and rank $r$. Write $\Gamma^{\prime}$ for a torsion-free normal subgroup of $\Gamma$ with $G=\Gamma / \Gamma^{\prime}$ finite. We apply Corollary 5.2 to $\Gamma / \Gamma^{\prime}$ with $r=\operatorname{rk}(\Gamma)$. We recover a $\mathbb{Z}[\Gamma]-$ complex $\mathbf{C}_{1} \otimes \cdots \otimes \mathbf{C}_{r}$ such that $H^{*}\left(\mathbf{C}_{1} \otimes \cdots \otimes \mathbf{C}_{r}\right)=$ $H^{*}\left(S^{n_{1}} \times \cdots \times S^{n_{r}}\right)$; with $\mathbf{C}_{1} \otimes \cdots \otimes \mathbf{C}_{r}$ a complex of $\mathbb{Z}[H]$-projective modules for all finite $H<\Gamma$. On the other hand, we have that the $\mathbb{Z} \Gamma$-complex $C_{*}(\mathfrak{E} \Gamma)$ is contractible. Therefore the complex $\mathbf{D}=C_{*}(\mathfrak{E} \Gamma) \otimes \mathbf{C}_{1} \otimes \cdots \otimes \mathbf{C}_{r}$ is such that $H^{*}(\mathbf{D})=H^{*}\left(S^{n_{1}} \times \cdots \times S^{n_{r}}\right)$.

Lemma 5.3 With the notation above, the complex $\boldsymbol{D}$ is $\mathbb{Z}[\Gamma]$-projective.

Proof The complex $C_{*}(\mathfrak{E} \Gamma)$ decomposes as a direct sum of permutation modules: $C_{*}(\mathfrak{E} \Gamma)=\oplus_{\sigma} \mathbb{Z}\left[\Gamma / \Gamma_{\sigma}\right]=\oplus_{\sigma} \mathbb{Z}[\Gamma] \otimes_{\mathbb{Z}\left[\Gamma_{\sigma}\right]} \mathbb{Z}$. Here $\sigma$ spans the cells of $\mathfrak{E} \Gamma / \Gamma$. Consequently $\mathbf{D}=\oplus_{\sigma}\left(\mathbb{Z}[\Gamma] \otimes_{\mathbb{Z}\left[\Gamma_{\sigma}\right]} \mathbf{C}_{1} \otimes \cdots \otimes \mathbf{C}_{r}\right)$, so that we only need to prove that $\mathbb{Z}[\Gamma] \otimes_{\mathbb{Z}\left[\Gamma_{\sigma}\right]} \mathbf{C}_{1} \otimes \cdots \otimes \mathbf{C}_{r}$ is $\mathbb{Z}[\Gamma]$-projective. Let $Q_{\sigma}$ be a graded $\mathbb{Z}\left[\Gamma_{\sigma}\right]$-module such that $\left(\mathbf{C}_{1} \otimes \cdots \otimes \mathbf{C}_{r}\right) \oplus Q_{\sigma}$ is $\mathbb{Z}\left[\Gamma_{\sigma}\right]$-free. We then have that $\left(\mathbb{Z}[\Gamma] \otimes_{\mathbb{Z}\left[\Gamma_{\sigma}\right]} \mathbf{C}_{1} \otimes \cdots \otimes \mathbf{C}_{r}\right) \oplus\left(\mathbb{Z}[\Gamma] \otimes_{\mathbb{Z}\left[\Gamma_{\sigma}\right]} Q_{\sigma}\right)=\mathbb{Z}[\Gamma] \otimes_{\mathbb{Z}\left[\Gamma_{\sigma}\right]}\left(\left(\mathbf{C}_{1} \otimes \cdots \otimes \mathbf{C}_{r}\right) \oplus Q_{\sigma}\right)$ is $\mathbb{Z}[\Gamma]$-free.

We summarize the main result of this subsection in the following:

Corollary 5.4 For a group $\Gamma$ with finite $v c d$ and $\operatorname{rk}(\Gamma)=r$, there exist a finite dimensional contractible complex $C_{*}(\mathfrak{E} \Gamma)$ and $r$ finite dimensional $\mathbb{Z}[\Gamma]$-complexes $\boldsymbol{C}_{1}, \ldots, \boldsymbol{C}_{r}$ such that $\boldsymbol{D}=C_{*}(\mathfrak{E} \Gamma) \otimes \boldsymbol{C}_{1} \otimes \cdots \otimes \boldsymbol{C}_{r}$ is a $\mathbb{Z}[\Gamma]$-projective complex with $H^{*}(\boldsymbol{D}) \cong H^{*}\left(S^{n_{1}} \times \cdots \times S^{n_{r}}\right)$.

\section{References}

[1] A Adem, Torsion in equivariant cohomology, Comment. Math. Helv. 64 (1989) 401411 MR998857 
[2] A Adem, Lectures on the cohomology of finite groups, from: "Interactions between homotopy theory and algebra", (L L Avramov, J D Christensen, W G Dwyer, M A Mandell, B E Shipley, editors), Contemp. Math. 436, Amer. Math. Soc. (2007) 317-334 MR2355780

[3] A Adem, J H Smith, Periodic complexes and group actions, Ann. of Math. (2) 154 (2001) 407-435 MR1865976

[4] D J Benson, Representations and cohomology, I: Basic representation theory of finite groups and associative algebras, Cambridge Studies in Advanced Math. 30, Cambridge Univ. Press (1991) MR1110581

[5] D J Benson, Representations and cohomology, II: Cohomology of groups and modules, Cambridge Studies in Advanced Math. 31, Cambridge Univ. Press (1991) MR1156302

[6] D J Benson, J F Carlson, Complexity and multiple complexes, Math. Z. 195 (1987) 221-238 MR892053

[7] KS Brown, Cohomology of groups, Graduate Texts in Math. 87, Springer, New York (1982) MR672956

[8] F X Connolly, S Prassidis, Groups which act freely on $\mathbf{R}^{m} \times S^{n-1}$, Topology 28 (1989) 133-148 MR1003578

[9] T tom Dieck, Transformation groups and representation theory, Lecture Notes in Math. 766, Springer, Berlin (1979) MR551743

[10] T tom Dieck, Transformation groups, de Gruyter Studies in Math. 8, de Gruyter, Berlin (1987) MR889050

[11] R M Dotzel, G C Hamrick, p-group actions on homology spheres, Invent. Math. 62 (1981) 437-442 MR604837

[12] D L Ferrario, Self homotopy equivalences of equivariant spheres, from: "Groups of homotopy self-equivalences and related topics (Gargnano, 1999)", (K-i Maruyama, J W Rutter, editors), Contemp. Math. 274, Amer. Math. Soc. (2001) 105-131 MR1817005

[13] B Hanke, The stable free rank of symmetry of products of spheres, Invent. Math. 178 (2009) 265-298 MR2545682

[14] A Heller, A note on spaces with operators, Illinois J. Math. 3 (1959) 98-100 MR0107866

[15] A G Ilhan, Obstructions for constructing G-equivariant fibrations, $\mathrm{PhD}$ thesis, Bilkent University (2011) arXiv:1110.3880

[16] M A Jackson, Rank three p-groups and free actions on the homotopy product of three spheres, in preparation

[17] M A Jackson, Qd( $p)$-free rank two finite groups act freely on a homotopy product of two spheres, J. Pure Appl. Algebra 208 (2007) 821-831 MR2283428 
[18] I Madsen, C B Thomas, C T C Wall, The topological spherical space form problemII existence of free actions, Topology 15 (1976) 375-382 MR0426003

[19] J Milnor, Groups which act on $S^{n}$ without fixed points, Amer. J. Math. 79 (1957) 623-630 MR0090056

[20] J-P Serre, Cohomologie des groupes discrets, from: "Prospects in mathematics (Proc. Sympos., Princeton Univ., 1970)", (F Hirzebruch, L Hörmander, J Milnor, J-P Serre, ZM Singer, editors), Ann. of Math. Studies 70, Princeton Univ. Press (1971) 77-169 MR0385006

[21] P A Smith, Permutable periodic transformations, Proc. Nat. Acad. Sci. U. S. A. 30 (1944) 105-108 MR0010278

[22] P A Smith, New results and old problems in finite transformation groups, Bull. Amer. Math. Soc. 66 (1960) 401-415 MR0125581

[23] M Suzuki, Group theory II, Grund. der Math. Wissenschaften 248, Springer, New York (1986) MR815926 Translated from the Japanese

[24] R G Swan, Periodic resolutions for finite groups, Ann. of Math. (2) 72 (1960) 267-291 MR0124895

[25] O Únlú, E Yalçin, Fusion systems and constructing free actions on products of spheres arXiv: $1006.5797 \mathrm{v} 1$

Department of Mathematics, University of British Columbia

Vancouver BC V6T 1Z2, Canada

michele@math.ubc.ca

Received: 11 January 2011 Revised: 11 July 2011 